\title{
Zuranolone-An Investigational Oral Neuroactive Steroid and Positive Allosteric Modulator of GABA Type A Receptors for Postpartum Depression and Major Depressive Disorder
}

Handan Gunduz-Bruce

Sage Therapeutics, Inc., Cambridge, MA, USA

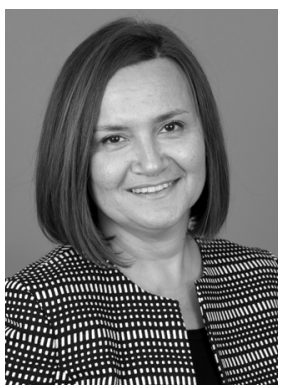

Handan Gunduz-Bruce

Handan Gunduz-Bruce is Senior Medical Director at Sage Therapeutics, Inc. and Assistant Clinical Professor of Psychiatry at Yale School of Medicine. She received her medical degree from the Istanbul Medical Faculty in 1991 and then completed her residency in psychiatry, followed by a fellowship in clinical neuroscience at the Long Island Jewish Medical Center of Albert Einstein College of Medicine. Earlier in her academic career, Dr. Gunduz-Bruce's research focused on the longitudinal course and pharmacological treatment of schizophrenia. Her later research included pathophysiology studies of schizophrenia and depression with a focus on the N-methyl-D-aspartate (NMDA) receptor function and GABAergic mechanisms using electrophysiological, imaging and biomarker approaches. More recently, she received her MBA degree in Leadership in Healthcare from Yale School of Management. Since the beginning of her tenure at Sage Therapeutics, Dr. Gunduz-Bruce has contributed to the approval of ZULRESSO ${ }^{\text {TM }}$, the first pharmacological treatment for postpartum depression in adults, and continues to oversee the development of zuranolone, an investigational oral neuroactive steroid and positive allosteric modulator of GABA, receptors, for postpartum depression and major depressive disorder.

\section{Keywords}

Zuranolone, SAGE-217, brexanolone, neuroactive steroid, neurosteroid, GABA positive allosteric modulator, postpartum depression, major depressive disorder

Disclosures: Handan Gunduz-Bruce is an employee of Sage Therapeutics, Inc. and holds stock options; in addition, Dr. Gunduz-Bruce is a named inventor on pending patent PCT Application Serial No. PCT/US2018/050012; NEUROACTIVE STEROIDS AND THEIR METHODS OF USE.

Review Process: This is an expert interview and as such has not undergone the journal's standard peer review process.

Compliance with Ethics: This is an expert interview and does not report on new clinical data, or any studies with human or animal subjects performed by the author.

Authorship: The named author meets the International Committee of Medical Journal Editors (ICMJE) criteria for authorship of this manuscript, takes responsibility for the integrity of the work as a whole, and has given final approval for the version to be published.

Access: This article is freely accessible at touchNEUROLOGY.com (C) Touch Medical Media 2020

Received: October 30, 2020

Published Online: December 23, 2020

Citation: US Neurology. 2020;16(2):81-3

Corresponding Author: Handan Gunduz-Bruce, Sage Therapeutics, Inc., 215 First St, Cambridge, MA 02142, USA. E: handan.gunduz-bruce@sagerx.com

Support: No funding was received in

the publication of this article.
1 Itered neurotransmission of $\gamma$-aminobutyric acid (GABA) has been implicated in the pathogenesis of depression. In this expert interview, Dr. Handan Gunduz-Bruce discusses the background and rationale for investigating zuranolone, an oral, positive allosteric modulator of GABA type $A\left(G_{A B A}\right)$ receptors, as a potential treatment for postpartum depression and major depressive disorder. She also provides an overview of the clinical programs that will determine zuranolone's place in the treatment paradigm for these depressive disorders.

\section{Q. What are neuroactive steroids and why are they being studied in depressive disorders?}

First I would like to briefly define the terms "neurosteroids" and "neuroactive steroids", since they are used interchangeably, but defined differently. The term "neurosteroid" refers to a class of endogenous steroids synthesized in the central nervous system (CNS) from cholesterol, which are potent modulators of the two major neurotransmitter systems - GABA and glutamate. "Neuroactive steroids" refers to endogenous molecules made in the body outside the CNS and also includes synthetic derivatives with actions similar to neurosteroids. ${ }^{1}$

The discovery of antidepressants in the 1950s, like many other medications, was serendipitous.,3 Monoamine oxidase (MAO) inhibitors, which were under investigation for the treatment of tuberculosis, demonstrated euphoric effects in the patients, paving the way for MAO inhibitors to be used as antidepressants. ${ }^{2,4}$ These observations were followed by the discovery of tricyclic antidepressants, and later, selective serotonin reuptake inhibitors, selective serotonin and norepinephrine, and atypical antidepressants. All these antidepressants target the monoamine system, namely serotonin, norepinephrine, or dopamine in different combinations.

Today, depression continues to be a major contributor to disability worldwide, which underscores a persistent unmet need. ${ }^{5}$ Additionally, the proposed underlying mechanism of depression has been expanded to implicate broader networks in brain function. 
One such hypothesis for depression implicates deficits in GABAergic networks. ${ }^{6}$ For example, reduced GABA levels have been observed in plasma, cerebrospinal fluid, and cortical brain tissues of patients with depression. There is also evidence for reduced expression of GABA-synthesizing enzymes in the brain tissue of people who have died by suicide, and a reduced number of GABAergic interneurons in the brain tissue of patients with depression. As GABA is the major inhibitory neurotransmitter in the brain, deficits in this network would theoretically be expected to lead to a wide array of dysregulation, including the monoaminergic system.

Going back to neurosteroids - they are synthesized from cholesterol in the brain. Even though neurosteroids have a steroid structure, they are distinct from glucocorticoids because they modulate ion channels, like GABA and glutamate receptors, and differ from steroids in their genomic effects as well.7.8 The prototypical neuroactive steroid that is a $\mathrm{GABA}_{A}$ receptor positive allosteric modulator is allopregnanolone (ALLO). An analog of ALLO is in development for postpartum depression and major depressive disorder based on its ability to potentially enhance inhibitory network activity through the GABA $A_{A}$ receptor, which may be compromised in these disorders, ${ }^{9}$ as discussed above.

\section{Q. What is the mechanism of action of allopregnanolone analogues in the treatment of postpartum depression?}

There are several proposed mechanisms of action of ALLO and its analogues in the treatment of postpartum depression, although the exact mechanism of action is not fully known. Firstly, it is important to recognize that ALLO is a positive allosteric modulator of both intra-synaptic and extra-synaptic GABA $_{A}$ receptors. ${ }^{1,10}$ This means that ALLO modulates both tonic and phasic inhibition in the brain. It is also important to note that ALLO plasma concentration increases substantially throughout pregnancy. Human data suggest that peak ALLO levels decline immediately after childbirth.11,12 Animal data suggest that the relatively sharp decrease surrounding childbirth in ALLO levels may be associated with the pathophysiology of postpartum depression. ${ }^{13}$

One proposed mechanism of action of ALLO in postpartum depression is based largely on animal studies. These studies show that there is down-regulation of $G_{A B A}$ receptors that parallel increasing ALLO levels during pregnancy, and this is followed by a recovery, namely up-regulation back to baseline levels, in the postpartum period. ${ }^{14}$ The hypothesized translation of these studies suggests that the emergence of severe depressive symptoms in women with postpartum depression may be due to an inability to up-regulate $\mathrm{GABA}_{\mathrm{A}}$ receptors quickly enough in the postpartum period.

More generally speaking, GABA neurotransmission has been implicated as one of multiple mechanisms in the pathophysiology of depression, based on findings of reduced GABA levels in plasma, cerebrospinal fluid, and cortical brain tissues of patients with depression, as well as by findings in post-mortem studies related to GABAergic markers. ${ }^{8}$ Therefore, because certain neurosteroids, like ALLO, are potent modulators of GABA, their pharmacologic effects would theoretically be expected to improve symptoms of depression based on these GABAergic deficits. ${ }^{1,6,10}$

Another proposed mechanism of action of ALLO in postpartum depression includes its effects on the hypothalamic-pituitary-adrenal axis dysfunction that have been implicated in depressive disorders, including postpartum depression. ${ }^{15}$

\section{Q. Brexanolone is your marketed product. What has been the impact of brexanolone on the treatment of postpartum depression and what are its limitations?}

Brexanolone injection is the first US Food and Drug Administration (FDA)-approved medication for the treatment of postpartum depression in adults. ${ }^{16}$ The mechanism of action of brexanolone in the treatment of postpartum depression is not fully understood, but is thought to be related to its positive allosteric modulation of $\mathrm{GABA}_{\mathrm{A}}$ receptors. Brexanolone injection is administered as a continuous intravenous infusion over 60 hours, so about 2.5 days, and was approved with a Risk Evaluation and Mitigation Strategy (REMS), under which healthcare facilities and pharmacies are required to be certified, and that patients are required to enroll in before administration of brexanolone injection. The goal is to mitigate the potential risk of serious harm resulting from excessive sedation and sudden loss of consciousness during the infusion. According to the REMS, brexanolone injection can only be administered at a certified healthcare facility where patients are monitored continuously for the duration of the infusion. Full details of the REMS, full Prescribing Information, and Boxed Warning can be found at zulressorems.com.

\section{Q. Could you give us an overview of the Landscape and Nest clinical programs?}

The Landscape program overarches our clinical studies with zuranolone, an investigational compound that is in development for the treatment of major depressive disorder. We have one completed phase II double-blind, randomized, placebo-controlled clinical study that met its primary endpoint in major depressive disorder. ${ }^{17}$ In a larger phase III, double-blind, randomized, placebo-controlled clinical study in adults with major depressive disorder, known as the MOUNTAIN Study (ClinicalTrials.gov identifier: NCT03672175), we studied two doses of zuranolone (30 mg and $20 \mathrm{mg}$ ) against placebo; however, this study did not meet its primary endpoint. ${ }^{18}$ Currently, we are studying a higher dose $(50 \mathrm{mg}$ ) in our phase III studies, including the WATERFALL Study (NCT04442490), another double-blind, randomized, placebo-controlled, clinical trial.

In addition, in an effort to collect "real-world" and longer-term data in a large number of patients, the SHORELINE Study (NCT03864614) has been enrolling subjects in an open-label design, allowing repeat administration of zuranolone as needed over 12 months. Lastly, we are interested in finding out whether co-initiation of zuranolone with a new antidepressant would improve depressive symptoms in patients with major depressive disorder. This is also a double-blind, randomized, placebo-controlled clinical trial, named the CORAL Study (NCT04476030), and plans are underway for its initiation this year. In these studies, we expect to collect both acute and repeat treatment data with longer-term follow-up using zuranolone.

In the Nest program for postpartum depression, the ROBIN Study (NCT02978326), which was a phase III double-blind, randomized, placebo-controlled clinical trial of zuranolone in patients with postpartum depression, met its primary endpoint. ${ }^{19}$ In addition, we have an ongoing phase III controlled clinical trial, called the SKYLARK Study (NCT04442503), which is currently enrolling in the USA and will expand to European sites in the near future. 


\section{Q. Where do you think zuranolone is likely to fit into the treatment paradigm for postpartum depression and major depressive disorder?}

As I mentioned previously, there are many, that is at least 25, approved antidepressants in the USA at this time. Depression remains a leading cause of disability with high cost to those who are suffering from it and also to society. The STAR*D study has demonstrated that response and remission rates are modest, and these rates further decrease as subsequent steps of antidepressants are administered..$^{20}$ So, all of these findings suggest that the unmet need in the treatment of depression continues.

In such an environment, zuranolone, once developed and if approved, may be a novel option for the treatment of postpartum depression and major depressive disorder in the future. We believe that if approved, zuranolone's duration of administration, of 2 weeks, may offer an important and valued treatment option for patients who suffer from these impactful conditions. $\square$
1. Zorumski CF, Paul SM, Izumi Y, et al. Neurosteroids, stress and depression: potential therapeutic opportunities. Neurose Biobehav Rev. 2013;37:109-22.

2. Pletscher A. The discovery of antidepressants: a winding path. Experientia. 1991:47:4-8.

3. Berton $\mathrm{O}$, Nestler EJ. New approaches to antidepressant drug discovery: beyond monoamines. Nat Rev Neurosci. 2006;7:137-51.

4. López-Muñoz F, Alamo C. Monoaminergic neurotransmission: the history of the discovery of antidepressants from 1950s until today. Curr Pharm Des. 2009;15:1563-86.

5. World Health Organization (WHO). Depression. 2020. Available at: www.who.int/news-room/fact-sheets/detail/depression (accessed December 9 , 2020).

6. Luscher $\mathrm{B}$, Shen $\mathrm{Q}$, Sahir $\mathrm{N}$. The GABAergic deficit hypothesis of major depressive disorder. Mol Psychiatry. 2011;16:383-406.

7. Rupprecht R, Holsboer F. Neuroactive steroids: mechanisms of action and neuropsychopharmacological perspectives. Trends Neurosci. 1999;22:410-6.

8. Rupprecht R, Reul JM, Trapp T, et al. Progesterone receptor-mediated effects of neuroactive steroids Neuron. 1993:11:523-30

9. Althaus AL, Ackley MA, Belfort GM, et al. Preclinical characterization of Zuranolone (SAGE-217), a selective Characterization of zu, Beto neuroactive sterid $G_{B B} A_{A}$.

modulator

10. Paul SM, Purdy RH. Neuroactive steroids. FASEB 1992;6:2311-22.

11. Hill M, Cibula D, Havlíková H, et al. Circulating levels of pregnanolone isomers during the third trimester of human pregnancy. J Steroid Biochem Mol Biol. 2007;105:166-75.

12. Gilbert Evans SE, Ross LE, Sellers EM, et al. 3alpha-reduced neuroactive steroids and their precursors during pregnancy and the postpartum period. Gynecol Endocrinol. 2005;21:268-79.

13. Maguire J, Ferando I, Simonsen C, Mody I. Excitability changes related to $\mathrm{GABA}_{\mathrm{A}}$ receptor plasticity during pregnancy. J Neurosc 2009;29:9592-601.

14. Maguire J, Mody I. GABA(A)R plasticity during pregnancy: relevance to postpartum depression. Neuron. 2008:59:207-13.
15. Payne JL, Maguire J. Pathophysiological mechanisms implicated in postpartum depression. Front Neuroendocrinol. 2019:52:165-80.

16. ZULRESSOTM (brexanolone) injection, prescribing information ver. 6/2019. Available at: https://assets.sagerx.com/zulresso/ prescribing-information.pdf (accessed November 5, 2020).

17. Gunduz-Bruce H, Silber C, Kaul I, et al. Trial of SAGE-217 in patients with major depressive disorder. N Eng/ J Med. 2019;381:903-11.

18. Sage Therapeutics, Inc. Press release. Sage Therapeutics reports topline results from pivotal phase 3 MOUNTAIN study of SAGE-217 in major depressive disorder. December 5, 2019. Available at: www.businesswire.com/news/ home/20191205005375/en/ (accessed December 9, 2020)

19. Sage Therapeutics, Inc Press release Sage Therapeutics announces SAGE-217 meets primary and secondary endpoints in phase 3 clinical trial in postpartum depression. January 7 , 2019. News release available at: www.businesswire.com/news/ home/20190107005377/en/ (accessed December 9, 2020).

20. Rush AJ, Warden D, Wisniewski SR, et al. STAR*D: revising conventional wisdom. CNS Drugs, 2009:23:627-4. 